\title{
Overexpression of the LKB1 gene inhibits lung carcinoma cell proliferation partly through degradation of c-myc protein
}

\author{
XUAN LIANG $^{1}$, KE-JUN NAN ${ }^{1}$, ZHAO-LUN LI $^{2}$ and QING-ZHI XU ${ }^{3}$ \\ ${ }^{1}$ Department of Oncology, First Affiliated Hospital; ${ }^{2}$ Department of Urology, Second Affiliated Hospital, \\ Xi'an Jiaotong University Medical College, Xi'an, Shaanxi 710061, ${ }^{3}$ Department of Radiation Toxicology \\ and Oncology, Beijing Institute of Radiation Medicine, Beijing 100850, P.R. China
}

Received November 25, 2008; Accepted January 5, 2009

DOI: $10.3892 /$ or_00000305

\begin{abstract}
LKB1 encodes a serine/threonine kinase generally inactivated in human lung cancers, which mediates cancer cell proliferation, migration and differentiation, but its biological function has not been completely elucidated. In this study, we demonstrated that LKB1 was associated with a substantial reduction of c-myc expression by using an inducible LKB1 expression system in the LKB1-null lung cell line A549. Nevertheless, the reduction of the c-Myc gene expression was not accompanied by corresponding reduction of mRNAs but protein, which can be abrogated by a proteosome inhibitor (MG132), suggesting that the reduction was associated with their increased degradation rather than transcriptional controls. Our results implied that the expression of c-Myc protein decreased by LKB1 in transfected cells may be a contributory factor in the process of cell proliferation. Overexpression of the LKB1 gene could inhibit the activation of ERK1/2 and STAT3 signaling pathways involved in the cell proliferation. Thus, LKB1-induced functional operation on c-Myc in promoting cell proliferation may occur in a novel mechanism, which may be regulated by ERK1/2 and/or STAT3 signal pathways in human lung carcinoma cells. Furthermore, our results give some insights into the understanding of how LKB1 inactivation contributes to lung carcinogenesis and emphasizes the central role played by LKB1 in lung cancer development.
\end{abstract}

\section{Introduction}

LKB1 (also termed as STK11) gene is deleted or mutated in many human cancers. The functional loss of LKB 1 or LKB1-inactivating mutations are known to be correlated with

Correspondence to: Dr Ke-jun Nan, Department of Oncology, First Affiliated Hospital, Xi'an Jiaotong University Medical College, Xi'an, Shaanxi 710061, P.R. China

E-mail: nankj1958@gmail.com

Key words: LKB1/STK11, c-Myc, lung carcinoma, cell proliferation, ERK1/2, STAT3 more than one-third of sporadic lung adenocarcinomas $(1,2)$, indicating that LKB1 gene inactivation is critical in lung cancer development, but its biological function has not been completely elucidated. The LKB1 protein has been shown to be involved in the inhibition of cell proliferation, which may participate in the regulation of cell growth in tumor cells by delaying the progression from G0-G1 to $\mathrm{S}$ phase of the cell cycle (3-6). The growth inhibitory effect in tumor cells is also mediated through the signaling of cytoplasmic LKB1 and/or the expression reduction of related proteins, such as p27, cyclin D and some transcription factors (7-9). The cellular Myc gene (c-Myc), which can initiate and maintain the transfected status of cells $(10,11)$, is one of the most frequently implicated target genes in carcinogenesis. Deregulated expression of the structurally unaltered Myc protein is sufficient to drive continuous cell proliferation and apoptosis in response to growth-promoting and growthinhibitory signals, respectively (12). Partanen and co-workers (13) have shown that disruption of epithelial cell organization by the lack of proper microenvironment or silencing of LKB1 could restore the ability of c-Myc to reinitiate the cell cycle and induce apoptosis. LKB1 might facilitate a functional operation on c-Myc that contributes to lung cancer development. However, there have been no published studies examining the direct relationship between c-Myc expression and LKB1 status in lung cancer cells.

To determine the effect of LKB1 on c-Myc expression, we overexpressed LKB1 in A549 lung cancer cells and simultaneously examined the changes of c-Myc expression. We further investigated whether the predominant effect of LKB1 gene on lung carcinoma proliferation was related to the degradation of c-Myc protein. c-Myc appears to affect intercellular signaling (14). In this study, we also examined some of cell proliferation associated signal transduction pathways in transfected cells stably expressing the LKB1 gene. With more LKB1 signaling pathways identified, a more profound understanding of the mechanisms that are associated to malignancies will give rise to the development of targeted cancer treatments. Collectively, we have a better understanding of the relationship between LKB1 and c-Myc, which can provide a novel molecular mechanism for the antitumor activity of LKB1 and may help further improve its effectiveness in controlling lung cancer development. 


\section{Materials and methods}

Cell culture and transfection. The LKB1-null human lung cancer A549 cells were plated in culture plates in Dulbecco's minimal essential medium (DMEM, Gibco, Grand Island, NY, USA) containing $10 \%(\mathrm{v} / \mathrm{v})$ fetal bovine serum (FBS, Gibco). The cultures were maintained at $37^{\circ} \mathrm{C}$ in a humidified atmosphere of $5 \% \mathrm{CO}_{2}$. After cultured up to $70 \%$ confluence on 6-well plates, cells were transfected with pcDNA-LKB1 or vehicle control pcDNA3.1 in the presence of Lipofectamine 2000 (Invitrogen Corp., Grand Island, USA) following the manufacturer's protocol. Transfection medium was replaced with growth medium containing 10\% FBS after cells were incubated with transfection reagents for $6 \mathrm{~h}$. Then, at day 16 after selection with complete DMEM containing $600 \mu \mathrm{g} / \mathrm{ml} \mathrm{G} 418$ (Gibco), all untransfected cells died and discrete clones were visible in transfected cells. These clones were expanded in the presence of $300 \mu \mathrm{g} / \mathrm{ml}$ G418 to be used for the further study. Both transfection and G418 selection were conducted under sterile conditions and duplicate plates were tested for each condition.

$R T-P C R$. Total RNA was isolated from the cells using TRIzol reagent (Invitrogen) according to the procedure provided by the manufacturer. Reverse transcription was performed from total RNA at $42^{\circ} \mathrm{C}$ for 60 min by M-MLV reverse transcriptase (RNase-free, Takara, Japan). PCR amplification was performed to synthesize c-Myc gene products. The forward/reverse primers were: c-Myc: 5'-TAG TGGAAAACCAGCAGCCT-3'/ 5'-CCTTACTTTTCCTTA CGCAC-3'; ß-actin: 5'-GGGAGAGCGGGAAATCGTGCG TGA-3'/ 5'-GATGGAGTTGAAGGTAGTTTCGTG-3'. The conditions included $94^{\circ} \mathrm{C}$ for $1 \mathrm{~min}$ and 30 cycles of $94^{\circ} \mathrm{C}$ for $15 \mathrm{sec}, 57^{\circ} \mathrm{C}$ for $15 \mathrm{sec}$, and $72^{\circ} \mathrm{C}$ for $30 \mathrm{sec}$, and the products were analyzed by electrophoresis on a $1.5 \%$ agarose gel.

Western blotting. Total cell lysates were prepared using the cell lysis buffer (Pierce, Rockford, IL, USA) following the manufacturer's instructions. Total protein was estimated using BCA analysis (Pierce). The samples were resuspended in SDS-PAGE loading buffer and heated at $95^{\circ} \mathrm{C}$ for $5 \mathrm{~min}$. Equal amounts of protein were loaded on gel and then transferred to nitrocellulose membrane (Mini-Protean and Trans-Blot systems, Bio-Rad Laboratories, Hercules, CA). The nitrocellulose membrane was blocked with $5 \%$ nonfat dry milk and separately incubated with corresponding specific primary antibodies, including 1:800 rabbit anti-LKB1 (Cell Signaling Tech, MA, USA), 1:1500 mouse anti-actin (Santa Cruz Biotechnology, Santa Cruz, CA), 1:800 mouse anti-phospho-p44/42-MAPK (Cell Signaling Tech), 1:800 mouse anti-total p44/p42-MAPK (Cell Signaling Tech) and 1:800 rabbit anti-phospho-STAT3 (Santa Cruz). After washing and incubating with corresponding secondary antibodies conjugated horseradish peroxidase (1:2000 dilution of goat anti-rabbit IgG antibody, 1:2000 dilution of goat anti-mouse $\operatorname{IgG}$ antibody, respectively) (Zhongshan reagent Biotechnology, Beijing, P.R. China). The proteins were detected by chemiluminescence and exposure to light-sensitive film.

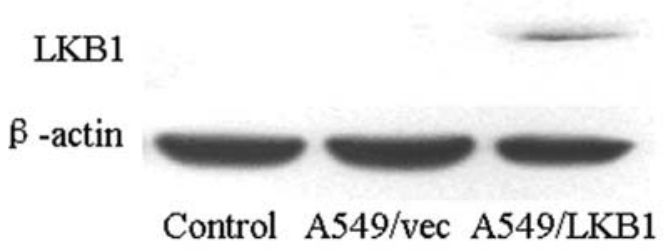

Figure 1. LKB1 expression in A549 cells transfected with pcDNA/LKB1 constructs or vehicle control pcDNA3.1. Control, untransfected cells; A549/vec, A549 cells transfected with the vehicle control pcDNA3.1; A549/LKB1, A549 cells transfected with target gene LKB1. B-actin was used as control for sample loading. These results are representative of at least 3 independent experiments.

Northern blot analysis. Total RNA was isolated using TRIzol (Invitrogen) according to the manufacturer's instructions. Northern blotting was performed using ${ }^{32} \mathrm{P}$-labeled cDNAs specific for c-Myc followed the manufacturer's instructions. The forward and reverse primers for PCR amplification of cDNA probes (Academy of Military Medicine, Beijing, P.R. China) were: 5'-TAGTGGAAAACCAGCAGCCT-3'; 5'-CCT TACTTTTCCTTACGCAC-3'. The glyceraldehyde-phosphate dehydrogenase (GAPDH) cDNA probe was used as the internal control for RNA sample loading.

Transient proteasome inhibition. In order to investigate the stability of c-Myc protein, we used the proteasome inhibitor MG132, which is widely used for the inhibition of ubiquitinmediated protein degradation, to analyze the c-Myc protein level after overexpressing LKB1 protein. The transfected cells were pre-treated with control medium and medium containing MG132 (1 $\mu \mathrm{mol} / \mathrm{l})$ for $4 \mathrm{~h}$, then the cells were incubated in control medium or MG132 $(25 \mu \mathrm{mol} / 1)$ at $37^{\circ} \mathrm{C}$ and $5 \% \mathrm{CO}_{2}$ for $24 \mathrm{~h}$. After incubation, cells were collected and examined for the expression of the c-Myc protein.

Cell proliferation assays. For sequential cell-counting experiments, cells were plated at 8,000 cells per well in 24-well plates in complete growth medium and incubated for $24 \mathrm{~h}$. Cells from three replicate wells were then counted (day 0) using a Hemacytometer (Beckman Coulter, CA). The remaining wells were treated and counted in triplicate at 24-h intervals for 7 days. For cell cycle analysis, cells were harvested following treatment with or without MG132 for $24 \mathrm{~h}$ and stained with propidium iodide (PI) according to the instruction of Cycle Test Plus DNA Reagent kits (Becton Dickinson, San Jose, CA). Samples were examined using flow cytometer (Becton Dickinson). For apoptosis analysis, double staining by FITC-Annexin V and PI was carried out as described by the manufacturer's instructions.

Statistical analysis. All data are reported as means \pm standard error (SE). Statistical significance of differences between mean values was assessed by Student's t-test for unpaired data using SPSS 13.0 statistical software (SPSS Inc., Chicago, IL, USA). Comparisons of data between multiple groups were performed with analysis of variance (ANOVA). $\mathrm{p}<0.05$ was considered statistically significant. 
A
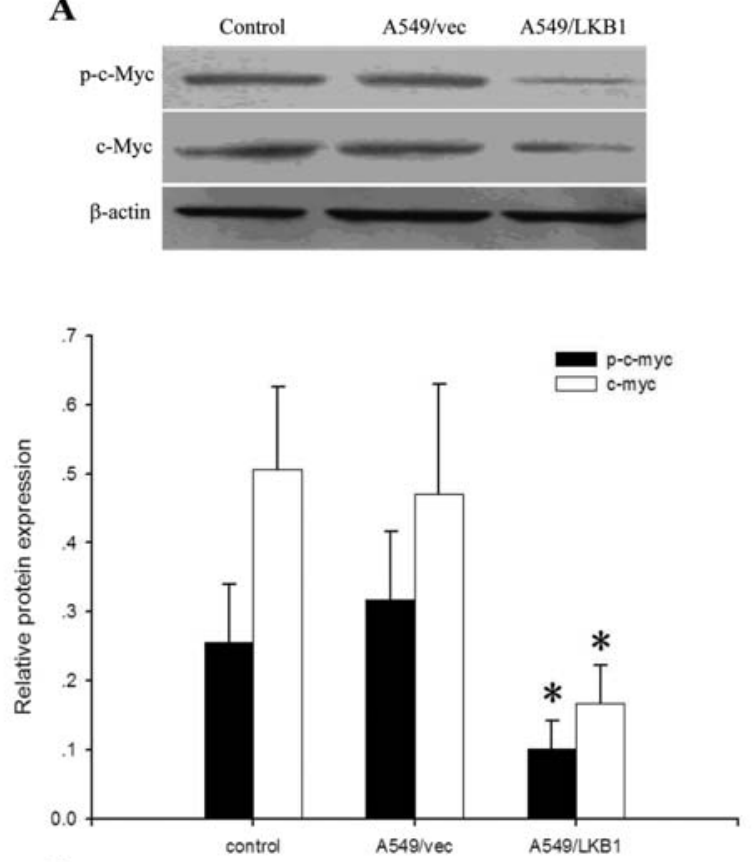

C

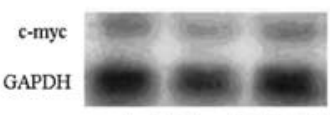

control A549/vec A549/LKB
B
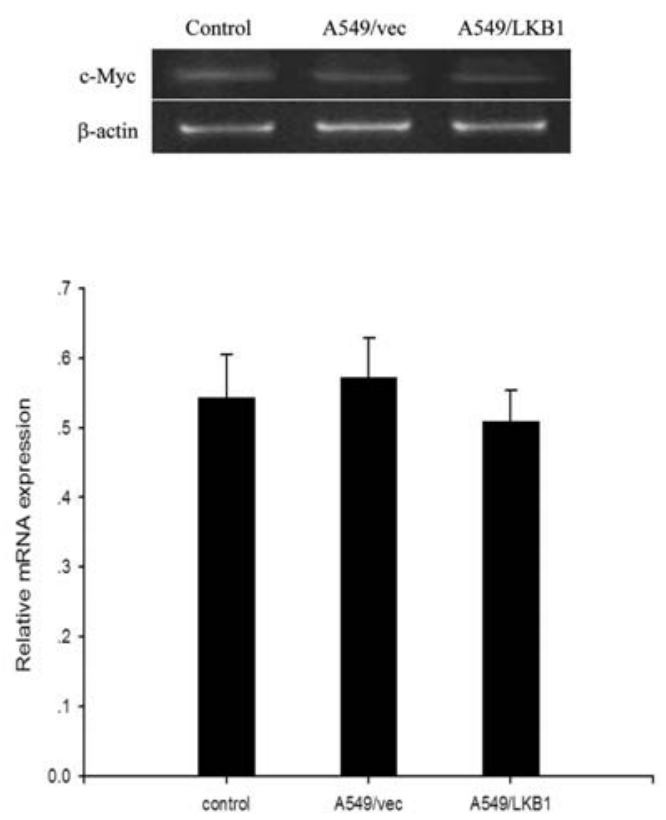

Figure 2. The expression of c-Myc in the transfected A549 cells. (A) Western blot analysis of c-Myc expression in A549 cells. Cells were collected and examined for expression of the c-Myc protein, $B$-actin was used as control for sample loading. Quantification of protein expression levels was expressed as the ratio of c-Myc/B-actin. The result is representative of 3 independent experiments ( ${ }^{*} \mathrm{p}<0.05$ vs. control). (B) RT-PCR analysis of c-Myc mRNA expression in A549 cells. (C) Northern blot analysis of c-Myc expression in A549 cells. Total RNA from the transfected cell lines was analyzed. Equal loading was confirmed by probe for GAPDH.

\section{Results}

Reintroduction of wild-type LKB1 into A549 cells induces the expression of $L K B 1$. Cells were transfected with a mammalian expression vector alone (pcDNA/Neo) and pcDNA/Neo containing the wild-type full-length cDNA of LKB. We selected transfected cells using the Neomicin/G418 resistance gene contained in the pcDNA/Neo expression vector. Sixteen days after selection with $\mathrm{G} 418$, there was a clear reduction in the number of colonies expressing wild-type LKB1 compared with those carrying the empty vector. To confirm that A549 transfected cells expressed exogenous LKB1, we performed Western blot experiments. LKB1 protein was detected in A549 cells transfected with exogenous wild-type LKB1, whereas no LKB1 protein expression was detectable in the cells transfected with the empty vector (Fig. 1).

Overexpressing LKB1 inhibits the expression of $c-M y c$ protein but not $m R N A$. As mentioned above, a significant decreased level of c-Myc protein was displayed in the transfected cells (Fig. 2A). To determine whether the downregulation of c-Myc by overexpressing LKB1 occurred at the transcription level and/or at the protein level, RT-PCR and Northern blot analysis of c-Myc expression was performed. As shown in Fig. 2B and C, overexpressing LKB1 did not alter the mRNA expression of the c-Myc gene. Thus, the downregulation of c-Myc by overexpressing LKB1 occurs at the protein level.
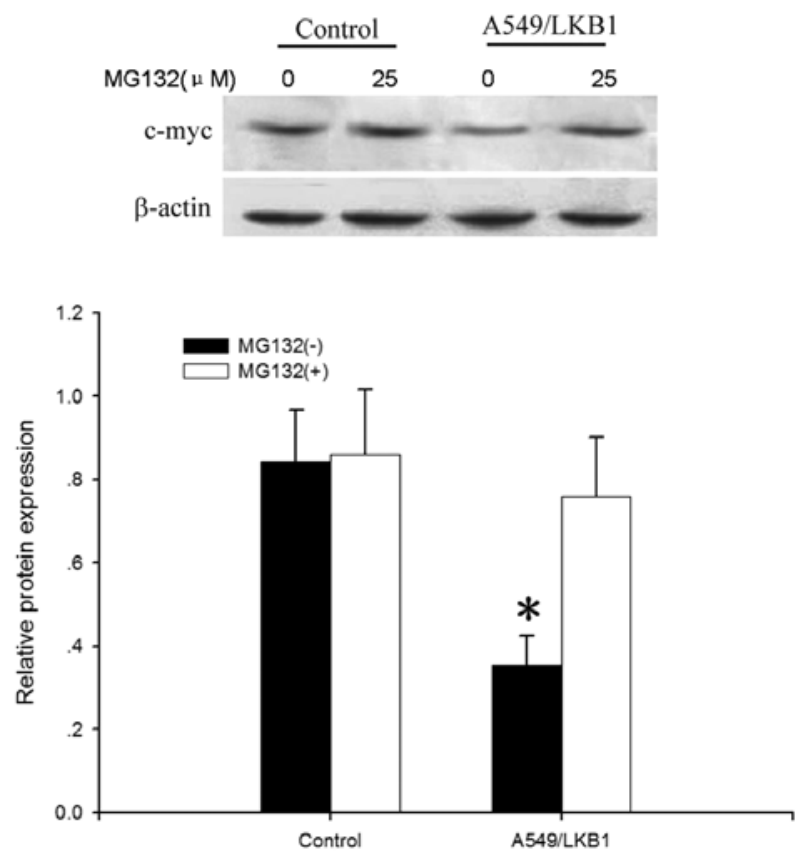

Figure 3. Effect of MG132 on expression of c-Myc in A549 cells overexpressing LKB1. Control, untransfected cells; A549/LKB1, A549 cells transfected with target gene LKB1. B-actin was used as control for sample loading. Quantification of protein expression levels was expressed as the ratio of $\mathrm{c}-\mathrm{Myc} / \mathrm{\beta}$-actin. The result is representative of three independent experiments $\left({ }^{*} \mathrm{p}<0.05\right.$ vs. control). 
A

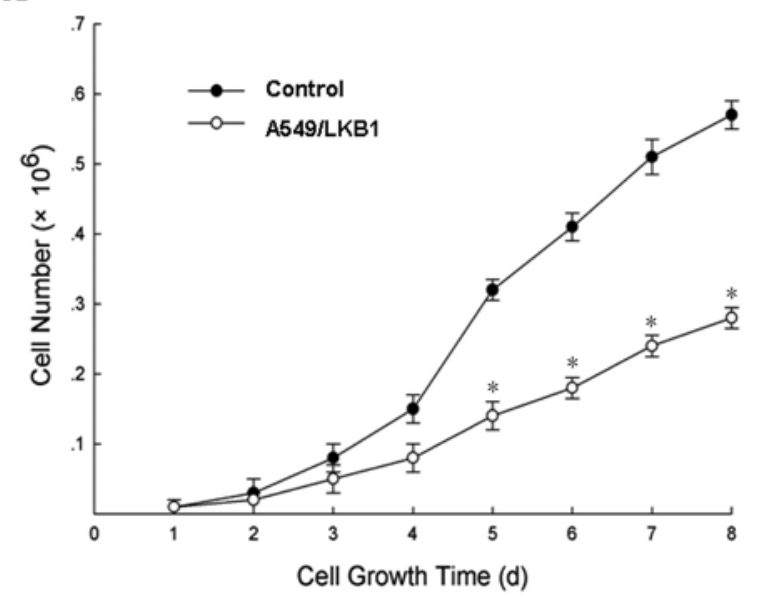

Figure 4. Effect of downregulation of c-Myc protein by LKB1 on A549 cell proliferation. (A) Growth curve of the A549 cell lines. Cells were grown in media containing G418 and the media were changed every two days. The number of cells was counted under hemocytometer. Data are expressed as mean \pm SD $\left({ }^{*} \mathrm{p}<0.05\right.$ vs. control). (B) Cell cycles analysis of the transfected cells and the control cells by flow cytometry, propidium iodide staining was performed and samples were analyzed. (C) Apoptosis analysis of the transfected cells and the control cells by bivariate FITC-Annexin V/PI flow cytometry. Each experiment was repeated at least 3 times.
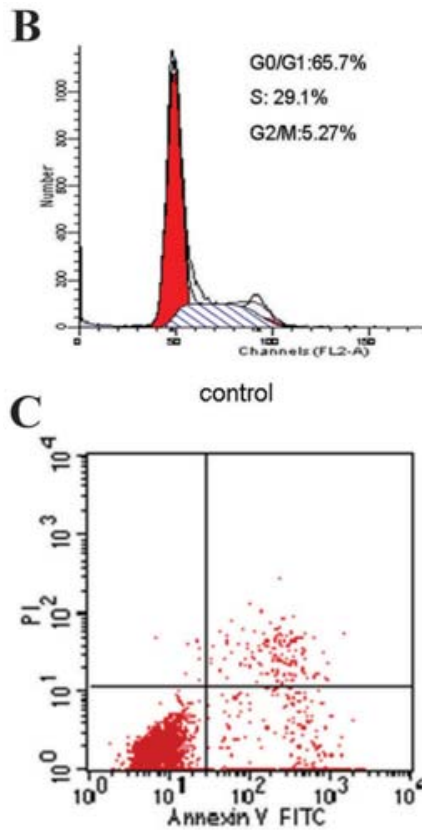

Control

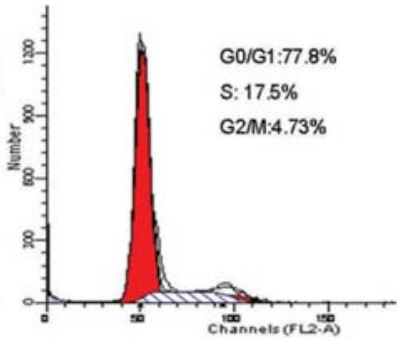

A549/LKB1

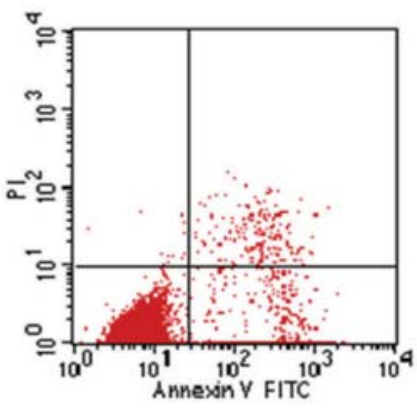

A549/LKB1

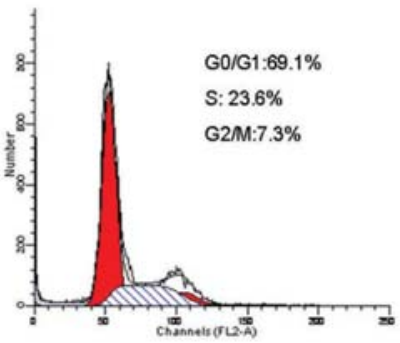

A549/LKB1+MG132

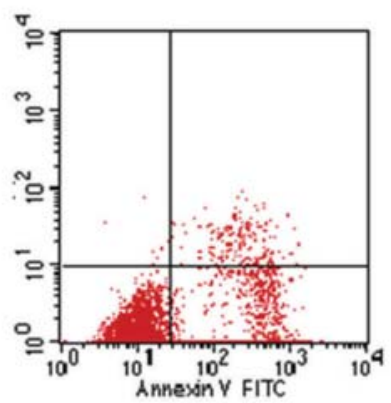

A549/LKB1+MG132
MG132 can restore the $c-M y c$ protein decreased by LKBI gene. c-Myc is a short-lived protein with a half-life of $\sim 30 \mathrm{~min}$ in many cells. To further confirm that c-Myc stability is regulated by LKB1, we also studied the effect of MG132, a specific inhibitor of proteasome, on the level of c-Myc. After treating cells with MG132 for $24 \mathrm{~h}$, we observed c-Myc protein expression by Western blotting. Fig. 3 shows that MG132 could restore the expression of c-Myc protein reduced by LKB1. Phosphorylated-c-Myc level obviously increased in lysate from cells treated with MG132 in comparison to control $(\mathrm{p}<0.01)$.

Downregulation of c-Myc protein by LKBI can inhibit cell proliferation. We examined the effect of c-Myc downregulation by ectopic expression of LKB1 on cell proliferation. The downward shift in the cellular growth curve was observed in the transfected A549 cells compared with control (shown in Fig. 4A). Cell cycle distribution evaluated by flow cytometry (Fig. 4B) showed an increase in the number of cells in the G1 phase and bivariate FITC-Annexin V/PI flow cytometric analysis showed significant apoptosis in transfected cells (Fig. 4C) $(\mathrm{p}<0.05)$. Moreover, treatment of A549/LKB1 cells with MG132 promoted the progression from G0-G1 to $\mathrm{S}$ phase and decreasing apoptosis.

LKB1 can influence the activities of MAPK and STAT signal pathways. The mitogen-activated protein kinases (MAPK) and signal transducer and activator of transcription 3 (STAT) pathways are important for cell proliferation. Malignant progression of tumor usually implies activation of intracellular pathways including MAPK/ERK and JAK/STAT3 pathways. To investigate whether the LKB1 gene could have any effect on these intracellular pathways, we analyzed the protein expression of p-ERK1/2 and p-STAT3 by Western blotting. The decreased expression of p-ERK1/2 and p-STAT3 was observed (Fig. 5).

\section{Discussion}

Like most tumor suppressor genes, LKB1 was identified because inactivation or germline mutations in the gene are associated with inherited cancer susceptibility $(2,15)$. Recent 

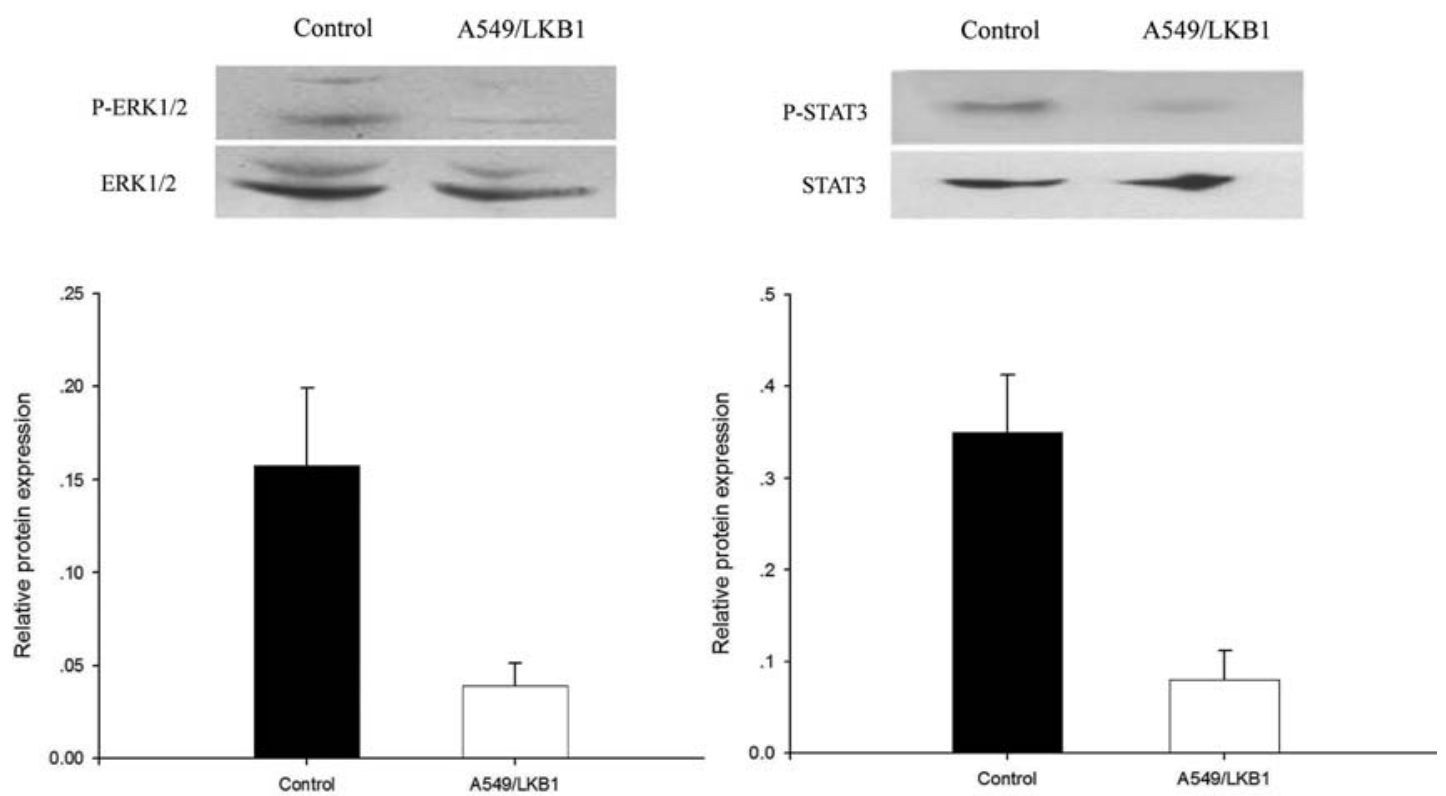

Figure 5. The expression of the proteins related with MAPK and the changes of tyrosine-phosphorylated STAT3 level in LKB1 transfected A549 cells determined by using Western blotting. Cells were harvested after cultured in serum-free medium for 24 h, lysates prepared, separated by SDS-PAGE and subjected to Western blotting for phospho-ERK1/2-MAPK, total ERK1/2-MAPK, phospho-STAT3 and total STAT3. Control, untransfected cells; A549/LKB1, A549 cells transfected with target gene LKB1 ( $\mathrm{p}<0.05$, Control vs. A549/LKB1).

data have revealed its novel functions, which provide new insight into the regulation of cell proliferation and metastasis (16-18). As a cellular negative regulator for growth and division, the tumor suppressor gene LKB1 plays an essential role in sensing various signals and serves as a focal point of signal integration to decide whether cells will undergo growth arrest or apoptosis. However, it is not clear how LKB1 gene triggers the significant cell-growth suppression and the relative molecules that contribute to lung cancer cell proliferation.

In the present study, we overexpressed LKB1 in A549 cells by means of an inducible LKB1 expression system. The cells transfected by pcDNA-LKB1 showed an extremely increased level of LKB1 protein. Interestingly, overexpressing the wild-type LKB1 in A549 cells triggered a clear downregulation of the c-Myc expression. For the first time, the direct relevance was found between c-Myc and LKB1 proteins acting in the same biochemical pathway in lung cancer cells. Moreover, overexpression of LKB 1 resulted in a remarkable depression on c-Myc expression at the protein level but not at the mRNA level. Furthermore, we used MG132, an inhibitor of protease, to examine whether it could restore the c-Myc level of transfected cells. As we expected, the expression of c-Myc protein was restored in the transfected cells. It suggested that this decreased expression was associated with the increased degradation rather than the transcriptional controls.

Deregulated c-Myc can lead to the alteration of cell cycle regulation as well as apoptosis. Some studies in vivo and in vitro have also shown that c-Myc is generally correlated with cellular proliferation and directly regulates those genes involved in cell cycle regulation (19-23). We propose that LKB1 represses the expression of c-Myc to inhibit the process of cell proliferation. Thus, apoptosis and

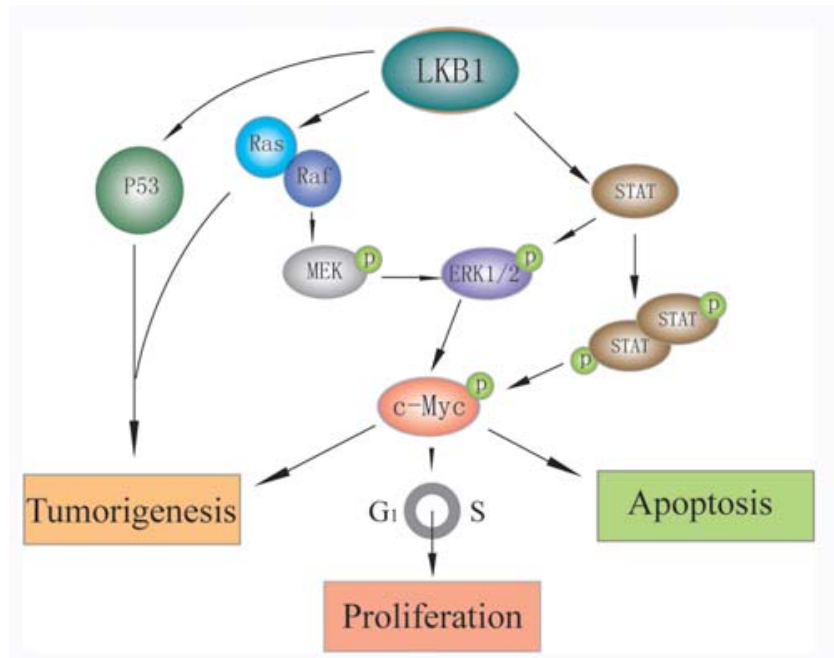

Figure 6. Schematic model of c-Myc regulation by LKB1. c-Myc signaling mediated cellular effects and their contribution to promote cell proliferation and tumorigenesis.

the cell-cycle analysis were performed in the present study. We found that downregulation of c-Myc by LKB 1 significantly contributes to the inhibition of G1-S transition, which maintained the A549 cells in the G0/G1 phase and induced apoptosis. Furthermore, we investigated whether the predominant effect of LKB1 gene on lung carcinoma cell proliferation was via degradation of c-Myc protein. In our results we found that restoring the c-Myc protein made the transfected cells undergo an extended growth phase prior to cell cycle entry and dereased apoptosis. Taken together, we suggest that loss of LKB1 is contributory to the expression of c-Myc protein, which might be mediated through a critical 
role in maintaining the stability of c-Myc protein. As there is a clear link between c-Myc and LKB1, we advocate that targeting LKB1 is an attractive strategy for developing new strategy and drugs for cancer therapy.

Tumor cells often show alteration in the signaltransduction pathways, leading to proliferation in response to external signals. Signaling pathways rely on protein phosphorylation ultimately leading to the activation of transcription factors that induce the expression of appropriate target genes (24-26). We have analyzed the activities of certain common signaling pathways that are known to be involved in cell proliferation. ERK1/2 signaling pathway has been implicated as a key regulator of cell growth. Another independent pathway, STAT3 signaling plays important roles in cell differentiation and proliferation $(27,28)$. Many cancer cells have constitutively activated MAPK, STAT-3 and thereby become insensitive to cell proliferation signaling. The A549 cell line reflect the intrinsic genetic characteristic, which has constantly activated RAS/RAF-1/MEK/ERK and JAK/STAT pathways. The expression of c-Myc could be elevated by constitutive activating STAT-3 signal and ERK1/2 also function as physiological c-Myc kinases to phosphorylate c-Myc (29). Blocking or downregulating ERK1/2 and STAT-3 signal could reduce c-Myc expression. In the present study, we examined the expression of phosphorylated STAT-3 (P-STAT3) and the ERK1/2 signaling pathway. LKB1 appears to be involved in regulation of multiple systematic signal pathways and acts as multifunctional protein. Our data demonstrate that overexpressing the LKB1 gene inhibits the activation of ERK1/2 and STAT3 proteins in the transfected A549 cells. Some studies demonstrated that one of the subtypes of MAPK could phosphorylate STAT3, which suggested cross-talk between MAPK cascades and JAK-STAT pathways $(24,30)$. It is also now clear that the cross-talk of these pathways contributes to the final proliferation effect on target cells by promoting G1-S phase cell cycle progression. Hence, the downstream molecules for mediating G1-S phase cell cycle progression may be concurrently regulated by these pathways to elicit the final physiological response. It has been suggested that c-Myc may integrate these signaling pathways (Fig. 6). Specifically, STAT3 regulates c-Myc production in many cells, identifying STAT-3 as a possible pathway of c-Myc induced carcinogenesis $(31,32)$. The MAPK pathway also affected c-Myc protein levels but to a lesser extent than the STAT3 pathway. These data suggest that the transfected A549 cell proliferation inhibited by LKB1 occurs as the result of a signal transduction pathway involving the activation of STAT3 and/or the ERK1/2 kinases.

In summary, we have demonstrated the growth suppression ability of ectopic LKB1 and its role in maintaining the stability of c-Myc protein in lung carcinoma cells. Downregulation of c-Myc by LKB1 significantly contributes to the inhibition of G1-S transition, which maintained the A549 cells in the G0/G1 phase and induced apoptosis. The antiproliferaion effect of LKB1 gene on lung carcinoma cells was partially via degradation of c-Myc protein, which further highlighted the relevance of LKB1 and c-Myc in lung cancer development. This critical information will allow us to have a better understanding of the function of LKB1 gene in lung cancer development and to choose the appro-priate target for therapeutic use.

\section{Acknowledgements}

This work was supported by National Natural Science Foundation of P.R. China (Grant No. 30270590).

\section{References}

1. Ghaffar H, Sahin F, Sanchez-Cepedes M, et al: LKB1 protein expression in the evolution of glandular neoplasia of the lung. Clin Cancer Res 9: 2998-3003, 2003.

2. Jimenez AI, Fernandez P, Dominguez O, et al: Growth and molecular profile of lung cancer cells expressing ectopic LKB1: down-regulation of the phosphatidylinositol 3'-phosphate kinase/PTEN pathway. Cancer Res 63: 1382-1388, 2003.

3. Sapkota GP, Kieloch A, Lizcano JM, et al: Phosphorylation of the protein kinase mutated in Peutz-Jeghers cancer syndrome, LKB1/STK11, at Ser431 by p90(RSK) and cAMP-dependent protein kinase, but not its farnesylation at Cys(433), is essential for LKB1 to suppress cell growth. J Biol Chem 276: 19469-19482, 2001.

4. Tiainen M, Ylikorkala A and Makela TP: Growth suppression by Lkb1 is mediated by a G(1) cell cycle arrest. Proc Natl Acad Sci USA 96: 9248-9251, 1999.

5. Tiainen M, Vaahtomeri K, Ylikorkala A, et al: Growth arrest by the LKB1 tumor suppressor: induction of p21 (WAF1/CIP1). Hum Mol Genet 11: 1497-1504, 2002.

6. Marignani PA: LKB1, the multitasking tumour suppressor kinase. J Clin Pathol 58: 15-19, 2005.

7. Xie X, Wang Z and Chen Y: Association of LKB1 with a WDrepeat protein WDR6 is implicated in cell growth arrest and p27(Kip1) induction. Mol Cell Biochem 301: 115-122, 2007.

8. Scott KD, Nath-Sain S, Agnew MD, et al: LKB1 catalytically deficient mutants enhance cyclin D1 expression. Cancer Res 67: 5622-5627, 2007.

9. Alessi DR, Sakamoto K, Bayascas JR: LKB1-dependent signaling pathways. Annu Rev Biochem 75: 137-163, 2006.

10. Grandori C, Cowley SM, James LP, et al: The Myc/Max/Mad network and the transcriptional control of cell behavior. Annu Rev Cell Dev Biol 16: 653-699, 2000.

11. Koch HB, Zhang R, Verdoodt B, et al: Large-scale identification of c-MYC-associated proteins using a combined TAP/MudPIT approach. Cell Cycle 6: 205-217, 2007.

12. Mundschau LJ and Faller DV: Platelet-derived growth factor signal transduction through the interferon-inducible kinase PKR: immediate early gene induction. J Biol Chem 270: 3100-3106, 1995.

13. Partanen JI, Nieminen AI, Makela TP, et al: Suppression of oncogenic properties of c-Myc by LKB1-controlled epithelial organization. Proc Natl Acad Sci USA 104: 14694-14699, 2007.

14. Krupitza G, Grusch M, Braun K, et al: TNFalpha-mediated cell death is independent of cdc25A. Cell Death Differ 5: 758-764, 1998.

15. Mehenni H, Lin-Marq N, Buchet-Poyau K, et al: LKB1 interacts with and phosphorylates PTEN: a functional link between two proteins involved in cancer predisposing syndromes. Hum Mol Genet 14: 2209-2219, 2005.

16. Qiu W, Schonleben F, Thaker HM, et al: A novel mutation of STK11/LKB1 gene leads to the loss of cell growth inhibition in head and neck squamous cell carcinoma. Oncogene 25: 2937-2942, 2006.

17. Boudeau J, Sapkota G and Alessi DR: LKB1, a protein kinase regulating cell proliferation and polarity. FEBS Lett 546: 159-165, 2003.

18. Katajisto P, Vallenius T, Vaahtomeri K, et al: The LKB1 tumor suppressor kinase in human disease. Biochim Biophys Acta 1775: 63-75, 2007.

19. Hermeking $\mathrm{H}$ and Eick D: Mediation of c-Myc-induced apoptosis by p53. Science 265: 2091-2093, 1994.

20. Liao DJ, Natarajan G, Deming SL, et al: Cell cycle basis for the onset and progression of c-Myc-induced, TGFalpha-enhanced mouse mammary gland carcinogenesis. Oncogene 19: 1307-1317, 2000.

21. Wierstra I and Alves J: The c-myc promoter: still MysterY and challenge. Adv Cancer Res 99: 113-333, 2008. 
22. Benassi B, Fanciulli M, Fiorentino F, et al: c-Myc phosphorylation is required for cellular response to oxidative stress. Mol Cell 21: 509-519, 2006.

23. D'Agnano I, Valentini A, Fornari C, et al: Myc down-regulation induces apoptosis in M14 melanoma cells by increasing p27(kip1) levels. Oncogene 20: 2814-2825, 2001.

24. Montag DT and Lotze MT: Rapid flow cytometric measurement of cytokine-induced phosphorylation pathways [CIPP] in human peripheral blood leukocytes. Clin Immunol 121: 215226, 2006.

25. Chang F, Steelman LS, Lee JT, et al: Signal transduction mediated by the Ras/Raf/MEK/ERK pathway from cytokine receptors to transcription factors: potential targeting for therapeutic intervention. Leukemia 17: 1263-1293, 2003.

26. Whitmarsh AJ: Examining signaling specificity to transcription factors. Methods 26: 217-225, 2002.

27. Klampfer L: The role of signal transducers and activators of transcription in colon cancer. Front Biosci 13: 2888-2899, 2008
28. Buettner R, Mora LB and Jove R: Activated STAT signaling in human tumors provides novel molecular targets for therapeutic intervention. Clin Cancer Res 8: 945-954, 2002.

29. Kaizu T, Ikeda A, Nakao A, et al: Protection of transplantinduced hepatic ischemia/reperfusion injury with carbon monoxide via MEK/ERK1/2 pathway downregulation. Am J Physiol Gastrointest Liver Physiol 294: G236-G244, 2008.

30. Inamura K, Matsuzaki Y, Uematsu N, et al: Rapid inhibition of MAPK signaling and anti-proliferation effect via JAK/STAT signaling by interferon-alpha in hepatocellular carcinoma cell lines. Biochim Biophys Acta 1745: 401-410, 2005.

31. Shirogane T, Fukada T, Muller JM, et al: Synergistic roles for Pim-1 and c-Myc in STAT3-mediated cell cycle progression and antiapoptosis. Immunity 11: 709-719, 1999.

32. Vougier S, Cheung SH, Li L, et al: Anomalous behaviour of the STAT3 binding site in the human c-myc $\mathrm{P} 2$ promoter. Biochem Biophys Res Commun 364: 627-632, 2007. 